\title{
The distribution of SNPs in human gene regulatory regions Yongjian Guo ${ }^{1,2}$ and D Curtis Jamison*1
}

Address: ${ }^{1}$ School of Computational Sciences, George Mason University, Manassas, VA 20110 USA and 2Virginia Bioinformatics Institute, Bioinformatics Facility I (0477), Virginia Tech, Blacksburg, VA 24060 USA

Email: Yongjian Guo - yguo@vbi.vt.edu; D Curtis Jamison* - cjamison@gmu.edu

* Corresponding author

Published: 06 October 2005

BMC Genomics 2005, 6:140 doi:10.1 I86/|47|-2164-6-140

This article is available from: http://www.biomedcentral.com//47/-2/64/6/140

(C) 2005 Guo and Jamison; licensee BioMed Central Ltd.

This is an Open Access article distributed under the terms of the Creative Commons Attribution License (http://creativecommons.org/licenses/by/2.0), which permits unrestricted use, distribution, and reproduction in any medium, provided the original work is properly cited.
Received: 16 March 2005

Accepted: 06 October 2005

\begin{abstract}
Background: As a result of high-throughput genotyping methods, millions of human genetic variants have been reported in recent years. To efficiently identify those with significant biological functions, a practical strategy is to concentrate on variants located in important sequence regions such as gene regulatory regions.
\end{abstract}

Results: Analysis of the most common type of variant, single nucleotide polymorphisms (SNPs), shows that in gene promoter regions more SNPs occur in close proximity to transcriptional start sites than in regions further upstream, and a disproportionate number of those SNPs represent nucleotide transversions. Additionally, the number of SNPs found in the predicted transcription factor binding sites is higher than in non-binding site sequences.

Conclusion: Current information about transcription factor binding site sequence patterns may not be exhaustive, and SNPs may be actively involved in influencing gene expression by affecting the transcription factor binding sites.

\section{Background}

Genetic variation has been found to be a ubiquitous phenomenon, and forms the genetic basis for species diversity. Currently, there are sequence variant data accumulated for humans [1], mouse [2], soybean [3] and other organisms. With the completion of Human Genome Project [4], the study of genetic variation has become one of the keystones in biomedical research, not only because it affects an individual's anthropometric characteristics but also because it influences risk of disease and response to environmental challenges [5]. The information derived from the study of variation not only deepens our understanding of human genes and evolution, but also brings benefits to the identification and treatment of human genetic diseases.
There are several different types of genetic sequence variants, including single nucleotide polymorphisms (SNPs), small deletion and insertion polymorphisms (indels), micro-satellite markers, and polymorphic insertion elements such as retrotransposons [6]. Because the most common variants are SNPs, the term is often abused as a synonym for genetic sequence variation. However, here we restrict its usage to the formal SNP definition: a single base change at a single position.

As a result of high-throughput genotyping methods, millions of human SNPs have been reported in recent years. To more efficiently study those with significant biological functions, a practical method is to concentrate efforts on SNPs located in genomic regions with important functions. There have been several studies focusing on 
Table I: Nucleotide substitution rate. Nucleotide substitution rates for SNPs in promoter regions $(P)$ and all SNPs genomewide $(A)$ show roughly similar rates for transition and transversion substitution, with a slight increase in CG transversions as would be expected from CG rich regions.

\begin{tabular}{lcrr}
\hline Type & Substitution & Frequency (P) & Frequency (A) \\
\hline Purine & $\mathrm{A} \leftrightarrow \mathrm{G}$ & $31.55 \%$ & $33.10 \%$ \\
Pyrimidine & $\mathrm{C} \leftrightarrow \mathrm{T}$ & $30.99 \%$ & $33.10 \%$ \\
Purine $\leftrightarrow$ Pyrimidine & $\mathrm{C} \leftrightarrow \mathrm{G}$ & $12.75 \%$ & $8.93 \%$ \\
Purine $\leftrightarrow$ Pyrimidine & $\mathrm{A} \leftrightarrow \mathrm{C}$ & $9.43 \%$ & $8.77 \%$ \\
Purine $\leftrightarrow$ Pyrimidine & $\mathrm{G} \leftrightarrow \mathrm{T}$ & $9.33 \%$ & $8.82 \%$ \\
Purine $\leftrightarrow$ Pyrimidine & $\mathrm{A} \leftrightarrow \mathrm{T}$ & $5.94 \%$ & $7.42 \%$ \\
\hline
\end{tabular}

evaluating how SNPs impact phenotype. For example, Ramensky et al. [7] have applied several rules to predict biological effects amino acid substitutions made by nonsynonymous coding SNPs. Clifford et al. [8] have explored non-synonymous coding SNPs effects on protein function through exploring those introducing amino acid alterations in protein motif regions.

Another genome region important to gene function are gene regulatory regions. Through binding of specific transcription factors, gene promoters are directly involved in gene transcription initiation and regulation. Thus sequence variation in gene promoters may alter transcription factor identification and binding, which in turn can influence gene expression and effect biological impacts. For example, it has been found that one allele of the HLAG gene (-725G), whose products inhibits maternal antifetal immune response, is highly associated with increasing risk for miscarriage [9]. One possible explanation is that the SNP falls within the binding site of interferon response factor-1 (IRF-1), affecting IRF-1 binding and down-regulating transcription of the HLA-G gene.

Here we analyze the distribution of SNPs in human gene regulatory regions. Putative transcription factor binding sites in the gene promoters were computationally derived and compared to previously identified SNPs. The results show that SNPs have differential distribution characteristics both in gene regulatory regions and in transcription factor binding sites when compared to the entire genomic SNP population.

\section{Results}

The build 33 release of the genome sequence contains 545 contig sequences mapped across all of the Homo sapiens chromosomes. 19,741 gene sequences were extracted from NCBI RefSeq database, which were linked to contig sequences by 15,803 LocusLink entries. While most loci have only one gene, some have two or more forming the structure of a gene cluster. In addition, sequence version discrepancies between the databases lead to the removal of approximately 3000 genes. The loss of these genes does not create any bias, as the errors in the affected sequence records were randomly distributed across the databases with no discernible pattern. Thus the final sequence data consisted of derived promoter regions for 16,429 genes.

TFD contains 3,749 mammalian transcription factor binding site sequences, with approximately 2,000 of them being found at least once in the derived gene promoter sequences. More than 1,700 binding sites were not found, presumably because they are either non-human, located outside the immediate upstream gene regions, or are binding sites for trans-regulatory elements. The number of predicted binding sites per promoter follows a normal distribution.

\section{SNP distribution in gene promoters}

Over 35,000 SNPs were found in the gene regulatory regions. More than $99.8 \%$ of them are two state alleles. The nucleotide substitution rates of promoter SNPs with two alleles are shown in Table 1. For comparison, substitution rates for all SNPs in the dbSNP database are also listed. In both sets of SNPs, transition class SNPs account for approximately two-thirds of the total number. This is not surprising, as the mismatches made by substitution within the same chemical group is thermodynamically more stable [10]. The remaining one-third SNPs are roughly evenly distributed across the four transversion types.

For SNPs in promoter regions, G/C substitution is higher than other nucleotide substitutions in the transversion type, which corresponds to the higher GC content in gene promoters. The distributions of SNPs of different types are plotted in Figure 1, which demonstrates that promoter SNPs are not evenly distributed in the promoter range of $[-2000,-1]$, with more SNPs are observed in the region close to the transcriptional start site. While the numbers of SNPs of all types are all increased in the region near transcriptional start site, the number of SNPs of transversion type shows the largest increase.

\section{Transcription factor binding site redundancy}

Data redundancy of transcription factor binding site motifs was evaluated by comparing binding site sequence similarity, which was divided into three categories: exact coverage, partial coverage and single nucleotide difference. The results of these three categories are shown in Table 2. There are 310 pairs of binding sites with exact sequence coverage such that the sequences can be represented using more generalized patterns. There are 1,114 pairs with partial coverage properties that can be regarded as a subsequence of other binding sites. Finally, 833 pairs are found with sequences that differ only at a single nucle- 


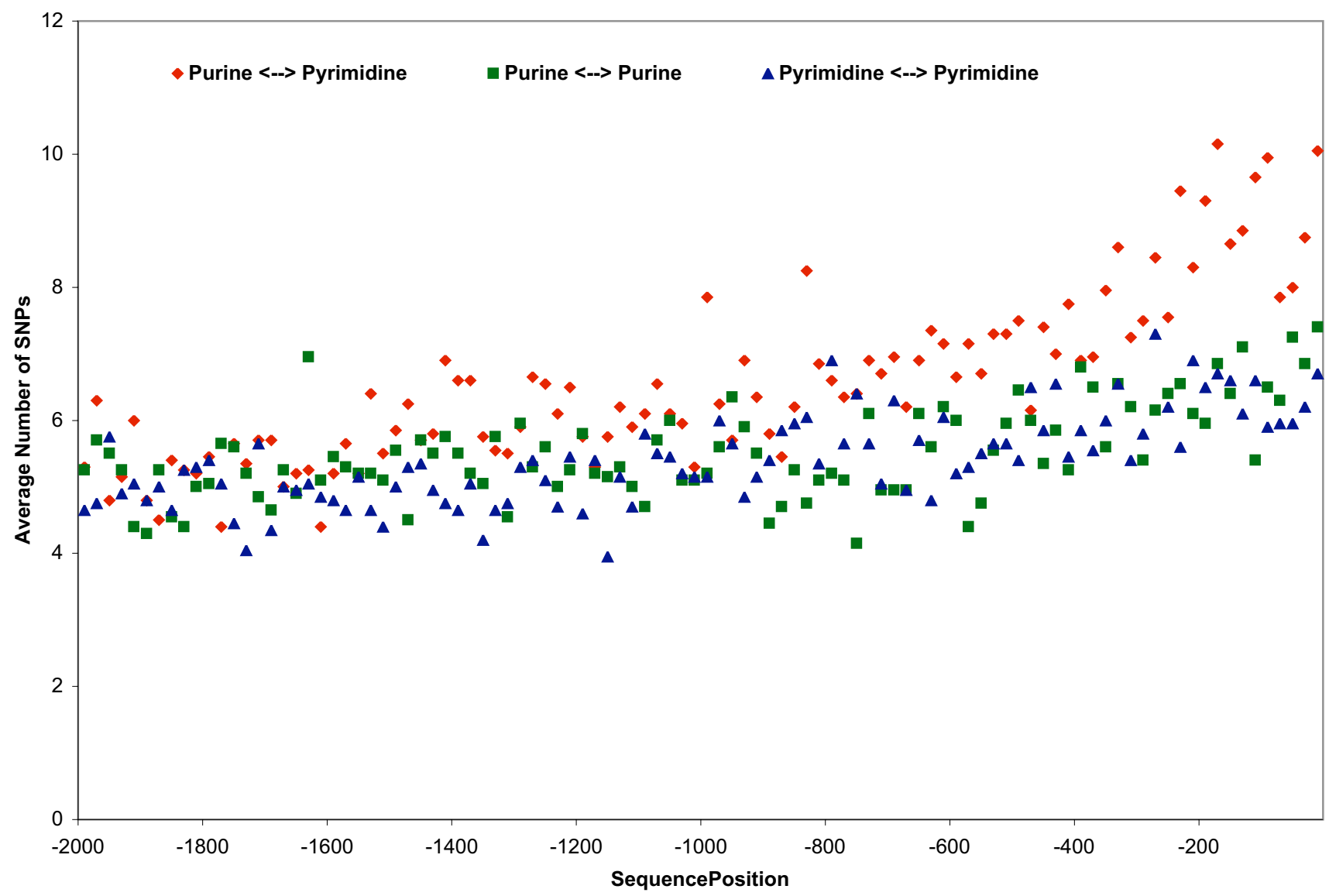

Figure I

Distributions of SNPs in different categories in gene promoter regions. Each symbol represents the average number of SNPs found in a 20 bp bin across approximately fourteen thousand promoter sequences with a full length of 2000 . This composite statistic shows the SNP density increases in proximity to the transcriptional start site.

otide position. Potential data redundancy of the binding site sequences makes it uninformative to study individual binding sites in the partial coverage and single nucleotide difference categories, so these motif pairs were merged into single motifs.

\section{Transcription factor binding sites distribution in gene promoters}

The distribution of binding sites in the gene promoter regions is not uniform, as shown in Figure 2. In the range of -2000 to -400 upstream the frequency is fairly constant. However the frequency increases in the regions closer to the transcriptional start site, reaching two to three times higher in the -100 to -50 range than that in the further sequence regions. This suggests most transcription factor binding sites occur within 250 bp of the initiation site.
Beyond the transcriptional start site the frequency of binding sites decreases dramatically, with the frequency value dropping below that of the entire upstream sequence.

Changing the expectation value from 0.1 to 0.01 causes the overall transcription factor binding site frequency to become smaller. The decrease is uniform across the entire sequence range of -2000 to 200 , as fewer binding site sequence patterns fulfilling the match criteria are detected. This uniformity suggests there is no issue of selective sensitivity.

Transcription factor binding sites in random sequence datasets simulating promoter sequence in the range of 2000 to -1 were predicted using an expect value 0.1 , and the results are shown in Figure 3 . There is a measurable 
Table 2: Transcription factor binding site sequence similarity. Examples of the three classes of degeneracy in binding site sequence motifs. For motif pairs with exact coverage, the less restrictive motifs were used. Motif pairs in the partial coverage and single difference categories were merged into a composite motif.

\begin{tabular}{lcll}
\hline Category & Pair Number & Binding Site Examples & \\
\cline { 3 - 4 } & & Sequence & Site Name \\
\hline Exact Coverage & & YYCCGCCC & EARLY-SEQI \\
& 310 & CCCCGCCC & $($ SpI)-TK.I \\
Partial Coverage & 1,114 & TGGICS3 & TGGCA_RS \\
Single Difference & 833 & YTGGCANNNTGCCAR & E-alpha_Y_box \\
& & TKCTGATTGTYTMM & NF-Y_CS \\
\hline
\end{tabular}

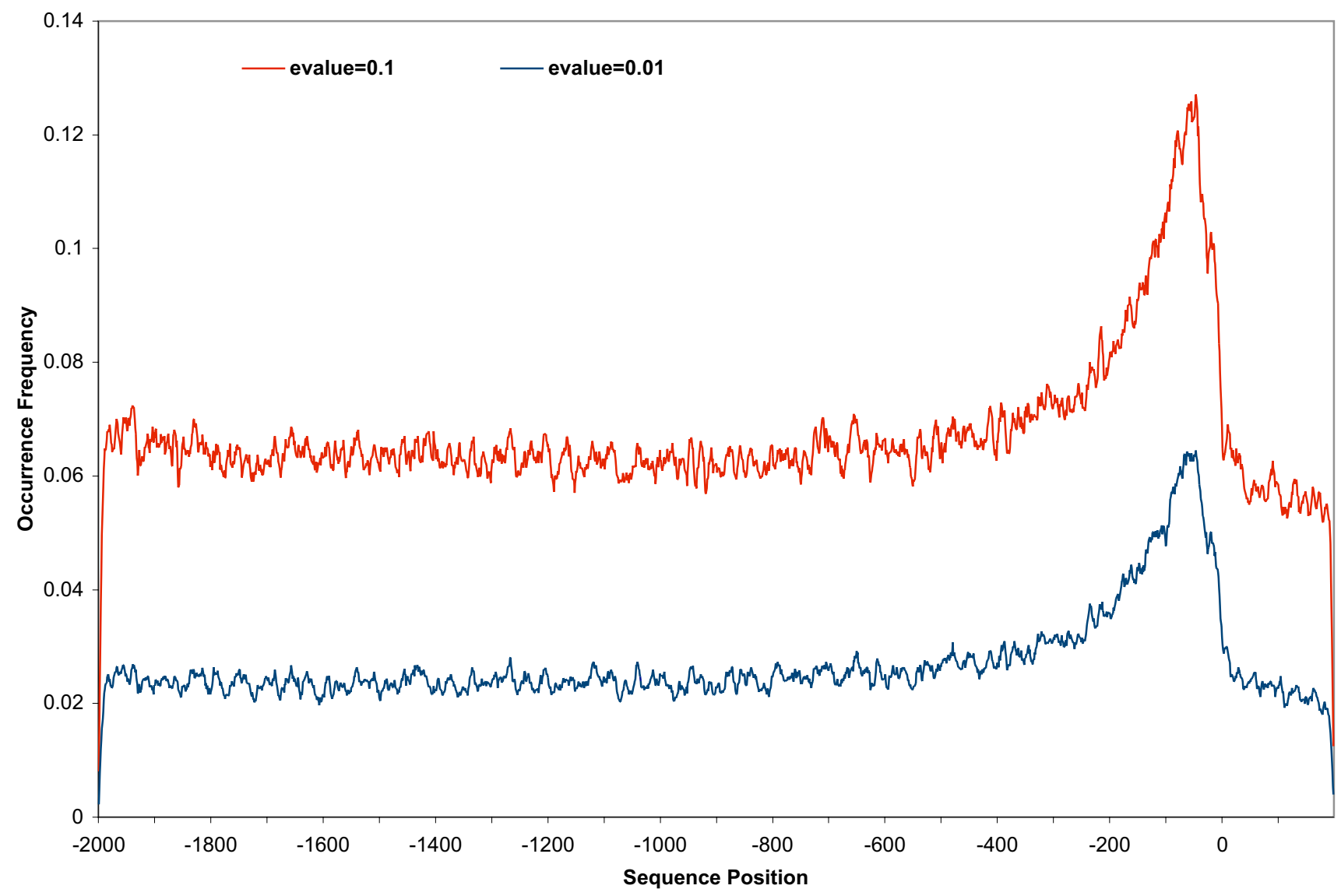

Figure 2

Distribution of transcription factor binding sites in gene promoter regions. The $x$-axis is the position index of gene promoter sequence, and the $y$-axis is the occurrence frequency of the binding sites across all promoters. Only genes with full length promoter sequences were included in calculation (approximately 14,000 sequences). A portion of the downstream sequence relating to transcriptional start site was also included. The position with index of 0 specifies the transcriptional start sites. 


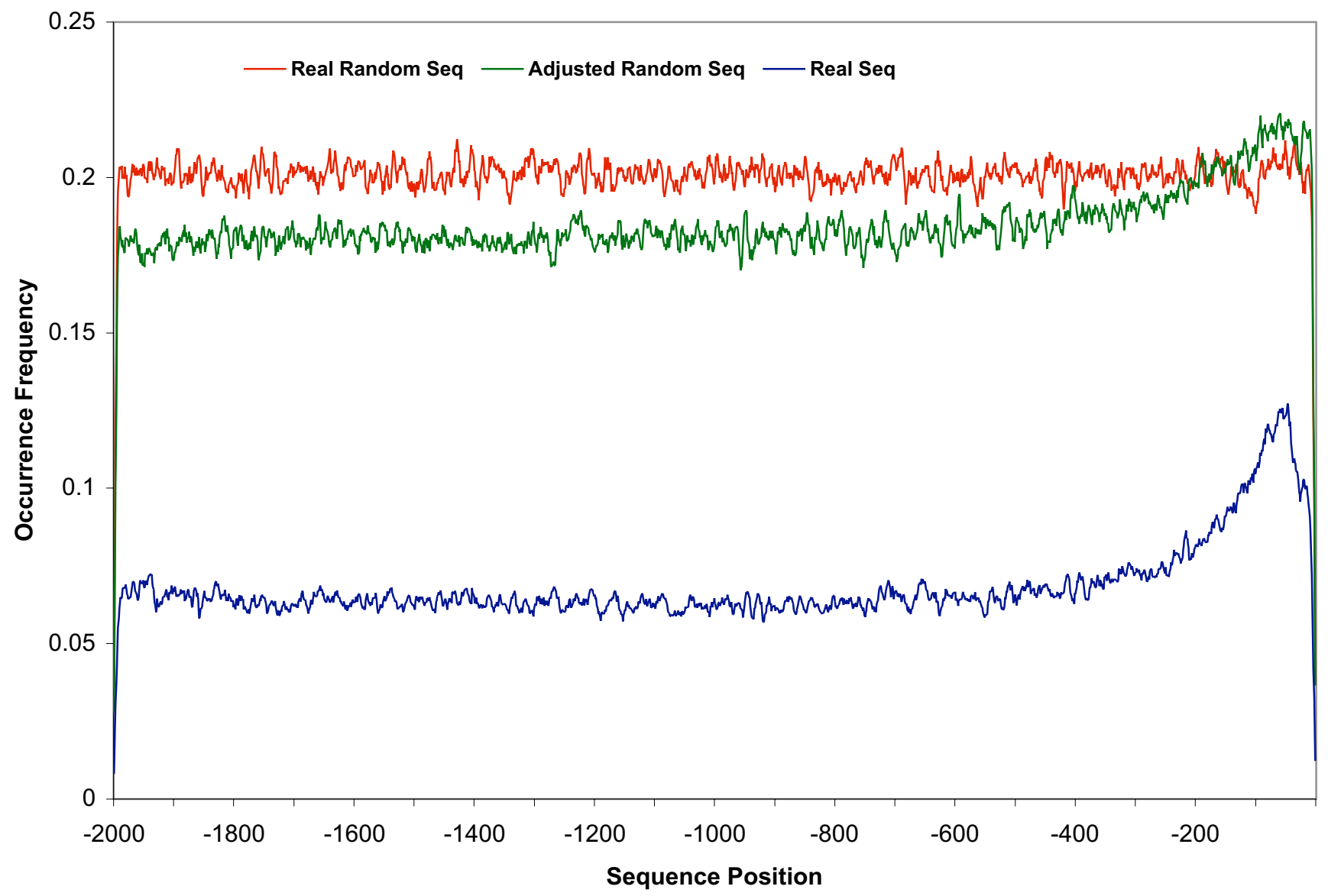

Figure 3

Comparison of transcription factor binding site distributions in random sequence datasets and real promoter sequence dataset. Each curve represents the occurrence frequency of predicted binding sites in different data sets of comparable size. "Real Random Seq" is a data set of completely random sequences in which the emission probabilities of A, C, G and T were equal and uniform across the entire $2 \mathrm{~kb}$. "Adjusted Random Seq" is a data set of random sequences generated with the adjusted emission probabilities of $A, C, G$ and $T$ according to that in the corresponding position at the real promoter sequence. "Real Seq" is the real promoter sequence dataset. An expectation value of 0.1 was used for detecting transcription factor binding sites in these datasets.

difference in the occurrence of transcription factor binding sites in the real promoter sequence dataset and the randomly generated sequence dataset. Three-fold more binding sites are detected in the random sequences than in the real promoter sequences, suggesting a non-random nucleotide distribution in real promoter sequences.

The patterns of transcription factor binding site occurrence in the two random sequence datasets are different. For completely random sequences, the occurrence appears to be flat across the whole range. However, in the frequency-based random sequences an increased appearance is observed in the sequence range close to transcriptional start sites similar to the pattern in the real promoter dataset. As the only difference between these two random datasets is the nucleotide emission probabilities at each sequence position, it suggests that transcription factor binding sites are biased by sequence composition. As GC content is enriched in the regions close to transcriptional start site [11], the fact that more transcription factor binding sites are observed in the promoter region near transcriptional start sites indicates that the binding site sequences are GC skewed.

\section{SNP distribution in transcription factor binding sites}

For 34,858 promoter SNPs mapped to 13,723 promoter regions, 2,078 (5.9\%) of them are located in the predicted transcription factor binding sites, and 1,969 (5.6\%) of 
Table 3: Promoter SNPs distribution on predicted transcription factor binding sites. Frequency of SNPs appearing in promoter regions and in putative transcription factor binding sites. The frequency of SNPs is significantly higher in putative binding sites than in the surrounding sequence $\left(p=2.2 \times 10^{-16}\right)$.

\begin{tabular}{lccc}
\hline Categories & SNPs & Nucleotides & Frequency \\
\hline Inside a binding site & 3,804 & $1,890,176$ & $0.20 \%$ \\
Outside the binding site & 31,054 & $25,555,824$ & $0.12 \%$ \\
Total & 34,858 & $27,446,000$ & $0.13 \%$ \\
\hline
\end{tabular}

them have alleles introducing new binding sites. The overlap of these two SNP sets has 243 members, indicating that their alleles may have different effects on transcription factor binding.

The nucleotide frequency of SNPs falling inside and outside transcription factor binding sites is shown in Table 3. The frequency of SNP distribution in the overall gene promoter region is $0.13 \%$. However, the frequency increases to $0.20 \%\left(\mathrm{p}=2.2 \times 10^{-16}\right)$ in the predicted transcription factor binding site regions.

A set of 293 experimentally derived transcription factor binding sites (associated with 85 genes) drawn from TRANSFAC were linked to predicted transcription factors used for this study. Of these, 13 contained SNPs, as shown in Table 4. The total number of nucleotides organizing the 293 TF binding sites is 5131. With 13 SNPs, the frequency of SNPs in the TF binding site sequence is $0.25 \%$, a value not significantly different from the frequency of promoter SNPs in the putative TF binding sites ( $\mathrm{p}$-value $=0.348$ ).

\section{Discussion}

Gene promoters for this study were derived using the human genome contig sequences and the RefSeq gene sequences, which are synthesis products of NCBI GenBank [12] records and other information sources. One concern is whether the gene sequences have reliable transcriptional start sites. The traditional experimental methods for identifying transcriptional start sites, such as S1 nuclease mapping [13], primer extension [14] and 5' RACE [15] are technically difficult and not always reliable. Consequently, the Eukaryotic Promoter Database [16], which collects the data from literatures, has only several hundred data entries.

In contrast, the Database of Transcriptional Start Sites [17] uses the full length cDNA library created by oligo-capping [18] to capture the longest mRNA transcript, and has accumulated more than 400,000 sequence entries. These cDNA sequences have been compared with the gene sequences in the RefSeq database to find the target genes and used to study the difference of the transcriptional start sites in the two datasets. According to DBTSS, for 8397 full length cDNA sequences whose corresponding RefSeq gene have been identified and compared, more than $85 \%$ of them have transcriptional start site differences equal to or less than 50 nucleotides. Thus the RefSeq gene sequences are relatively reliable in defining the transcriptional start sites.

Although more than $85 \%$ of RefSeq gene sequences have almost exact transcriptional start sites, it is possible that some of the derived upstream sequences may not be real gene promoters. The impact of false promoters on the observations is minimal however, as the statistics are calculated using composite statistics. The use of composite statistics also reduces potential bias introduced by highly studied genes, where there might be a greater number of SNPs simply due to a greater number of sequenced individuals, and bias due to the greater interest in the 200 bp immediately upstream of the transcription start site. The normal distribution of binding sites per promoter also argues against any bias due to frequently studied genes, because if there were such study bias we would expect a bimodal distribution with the frequently studied genes having more SNPs forming one mode, and the less studied genes with fewer SNPs forming the second.

Transcription factor binding site prediction algorithms tend to over-predict sites. However, correlation of experimentally determined sites with the predicted sites showed no significant deviation in the number of SNPs falling within confirmed or predicted transcription factor binding sites. The nucleotide frequency of SNPs in experimental transcription factor binding site sequence is $0.25 \%$, which is comparable with the $0.20 \%$ nucleotide frequency of promoter SNPs in the putative TF binding sites, and significantly higher than the $0.13 \%$ overall nucleotide frequency in the promoter sequences used in this study. Thus we are reasonably certain that the observations reflect a real phenomenon.

When transcription factor binding sites are mapped on gene promoter regions, the anchoring transcriptional start site forms a separating point as shown in Figure 2. 
Table 4: Promoter SNPs distribution on experimental transcription factor binding sites. Genes which have SNPs in experimentally determined transcription factor binding sites. The frequency is not significantly different from the frequency of promoter SNPs in the putative TF binding sites $(\mathrm{p}$-value $\mathbf{=} \mathbf{0 . 3 4 8})$.

\begin{tabular}{|c|c|c|c|c|c|c|}
\hline $\begin{array}{c}\text { Gene Accession } \\
\text { ID }\end{array}$ & $\begin{array}{l}\text { SNP Accession } \\
\text { ID }\end{array}$ & $\begin{array}{l}\text { SNP } \\
\text { Position }\end{array}$ & $\begin{array}{l}\text { TF Binding } \\
\text { Site Start }\end{array}$ & $\begin{array}{c}\text { TF Binding Site } \\
\text { End }\end{array}$ & Binding Site Name & $\begin{array}{l}\text { TRANSFAC Binding } \\
\text { Site ID }\end{array}$ \\
\hline NM 000384 & rs9282608 & -149 & -155 & -134 & ApoB-site [25] & R03692 \\
\hline NM 000384 & rs13306199 & -82 & -86 & -61 & AF-I-k-apoB [26] & $\mathrm{R} 01612$ \\
\hline NM 005252 & rs4645852 & -84 & -85 & -78 & c-fos.5 [27] & R00470 \\
\hline NM 002467 & rs4645940 & -1827 & -1834 & -1820 & c-myc-PUR [28] & R0430I \\
\hline NM 002467 & rs|3250910 & -322 & -326 & -310 & NHE [29] & R0I804 \\
\hline NM 002690 & rs2307I58 & -83 & -93 & -75 & SpI-human-beta-polymerase [30] & $\mathrm{R} 00287$ \\
\hline NM 002690 & rs2307I55 & -55 & -72 & -51 & CREB-beta-polymerase [3I] & $\mathrm{R} 00288$ \\
\hline NM 000805 & rs988955I & -100 & -102 & -95 & gastrin-negative-element [32] & R0203I \\
\hline NM 000176 & rs 10482604 & -708 & -713 & -691 & GRFE [33] & R0330I \\
\hline NM 000600 & rs|3447445 & -64 & -80 & -64 & NF-kappaB-IL-6 [34] & R0I634 \\
\hline NM 000208 & rs 1864010 & -494 & -511 & -486 & SpI-insulin_receptor [35] & $\mathrm{R} 03287$ \\
\hline NM 002123 & rs285427I & -106 & -117 & -99 & HLA-DQBI-Xbox [36] & $\mathrm{R} 03695$ \\
\hline NM 001063 & rs8177185 & -617 & -623 & -599 & transferrin-undefined-site [37] & $\mathrm{R} 01453$ \\
\hline
\end{tabular}

Upstream of the transcriptional start site, occurrence frequency of binding sites goes up, whereas the frequency is reduced downstream of the start site. It is clear that SNP distribution in gene promoter sequences is not even. SNPs occur more often in regions close to transcriptional start site than further regions, as is shown Figure 1. This conforms to other observations that the SNP distribution in human chromosomes is not uniform [19].

In general, SNP frequency is directly related to the evolutionary pressure on the target genome regions. For example, more SNPs are accumulated in repetitive sequence, introns and pseudogenes, as the evolution pressure in these regions is relatively low compared to functional gene sequence regions. Given this hypothesis, it is difficult to explain why more SNPs are observed in the sequence region close to transcriptional start site, as the region is important to the initiation of gene transcription, and sequence alteration has potentials to influence gene expression.

One possible explanation is that higher SNP frequency is related to important functions of the regions close to transcription start sites. The accumulation of SNPs in the human genome is like a snapshot of human evolutionary history in which genes, especially those with specific functions, are under continuous natural selection pressure and alteration by mutation, genetic drift and gene flow. As a result, the expression pattern of a gene may be changed. While some genes become totally inactive, others experience expression level alteration. It is possible that SNPs occurring in gene promoter regions play an important role in such scenario, so that the higher frequency of SNPs close to transcriptional start site is related to subtle alteration of gene expression which results in population diversity.
Nucleotide changes caused by SNPs can be classified as transitions and transversions. Generally, transitions are more conservative, because the substituted nucleotides belong to the same chemical group, purine or pyrimidine. In contrast, SNPs resulting in transversions involve nucleotide substitution across purine and pyrimidine chemical groups. The different chemical structures normally limit the occurrence of SNPs of transversion type, however such SNPs have increased frequency in regions close to transcriptional start site as shown in Figure 1. Comparison of nucleotide substitution rates for SNPs in gene promoter regions versus the entire genome also demonstrates that transversion SNPs are increased in promoter regions. It is notable that SNPs with C/G substitution are significantly increased, suggesting that SNPs with unfavorable structure substitution are indeed selected for in the gene promoter regions close to transcriptional start site.

One explanation for the increased SNPs of transversion type may lie in the gene transcription mechanism. During gene transcription, the DNA double helix needs to be opened so that one strand can be used as the template for producing mRNA. In the DNA double helix structure, there are two hydrogen bonds between $\mathrm{A}$ and $\mathrm{T}$ base pairs and three hydrogen bonds between $\mathrm{G}$ and $\mathrm{C}$ base pairs, thus opening a G-C base pair requires more energy than an A-T base pair opening does. GC-rich regions often require formation of protein-DNA complexes to facilitate helix opening. Involvement of proteins allows tighter control mechanisms on gene transcription so that gene expression can more accurately respond to environmental conditions. An increased frequency of SNPs with C/G substitutions may be evolutionarily selected for to maintain higher GC content and allowing changes of transcription regulation based on sequence alteration. 
It is curious that there are more SNPs in binding site regions than in normal sequence regions. This is contradictory to the hypothesis that transcription factor binding sites are highly conserved because they are important to gene transcription regulation. It is possible that the observations are an artifact and the identified putative transcription factor binding sites are not real binding sites for human genes. As the binding site sequence patterns used are in the mammalian category of the TFD database, some transcription factor binding sites may belong to organisms other than human. However, the false positive binding sites would be simply normal sequence regions in gene promoter which should have the lower genomic SNP frequency, biasing the frequency downward rather than upward.

A second potential artefact is quality control in the transcription factor binding site data used. While data redundancy may exist as shown in Table 2, it is possible that some binding site sequence patterns are not exhaustive. Thus, identified SNPs in the binding sites may have no influence on the binding site integrity and therefore will not affect transcription factor binding and gene expression. This hypothesis cannot be ruled out computationally and will require experimental results to confirm if a SNP found in a transcription factor binding site can exert a real effect on gene expression. If not, the sequence pattern of the binding site needs to be expanded. However, the sequence patterns used for prediction represent consensus sequences drawn from experimental data, and are probably relatively stable and exhaustive.

A third explanation for increased observation of SNPs in the binding sites is that SNPs are involved in altering gene expression during evolution through affecting the binding site sequences. Effects of SNPs in the binding site is likely not simple. While some binding sequence changes made by SNPs may totally interrupt gene expression, others may only influence the level of expression. Considering that gene transcription is a complex process involving many transcription factors, a single position change may not influence all of them. In evolution, the requirement for adjusting certain gene expression level to certain environmental factors forms a natural selection on gene regulation, on which SNPs occurring in transcription factor binding site have direct effect. Therefore, the fact that more SNPs are in the binding sites may demonstrate diverse requirements for different gene expression under different condition.

\section{Conclusion}

A biological explanation for increased observation of SNPs in the binding sites may be that SNPs are involved in altering gene expression during evolution by affecting the binding site sequences. The effects of SNPs in the binding site is likely not simple. While some binding sequence alterations made by SNPs may totally interrupt gene expression, others may only influence the level of expression. Considering that gene transcription is a complex process involving many transcription factors, a single position change may not influence all of them.

In evolution, the requirement for adjusting certain gene expression level to certain environmental factors forms a natural selection on gene regulation, on which SNPs occurring in transcription factor binding site have direct effect. Therefore, the fact that more SNPs are in the transcription factor binding sites may demonstrate diverse requirements for different gene expression under different condition. Thus, promoter SNPs may be an active factor in natural selection on gene transcription.

\section{Methods}

Data from four different sources (LocusLink, Genome, TFD, and dbSNP) were used to generate the promoter SNP data set. At each step, a number of steps to clean and normalize the data were undertaken. Figure 4 shows the general steps and data sources used in our investigation.

\section{Gene promoter identification}

Gene promoter sequences were calculated using human genome contig sequences (build 33) from NCBI and gene location information from LocusLink. For single gene loci, the gene was mapped onto its target contig using the scope value, reverse complementing the contig sequence as necessary. The $2 \mathrm{~kb}$ sequence immediately upstream of the transcriptional start site was then extracted and used as the promoter sequence. For genes located closer than $2 \mathrm{~kb}$ to the start of contig sequence, the length of the extracted upstream sequence was limited to the available sequence. Loci having more than one gene suggest that genes are cotranscribed, or gene products resulting from alternative splicing. Therefore, the start site of the first gene in the locus was used to derive the upstream sequence, which was used as the promoter sequence for all of the genes in the locus. The few conflicts from data discrepancies across different NCBI databases were resolved by forcing agreement on sequence version in the data extraction pipeline.

\section{SNP distribution in gene promoters}

Promoter SNPs were mapped using the SNP location information from dbSNP [6]. Non-unique and loosely mapped SNPs accounted for only $5.5 \%$ for the total number of SNPs and were excluded. SNPs were then classified according to the substitution type as either transitions, which includes nucleotide substitution within the purine $(\mathrm{A} / \mathrm{G})$ or pyrimidine $(\mathrm{C} / \mathrm{T})$ group, or as transversions, which represents substitution across the groups. The general distribution pattern of SNPs was then computed. 


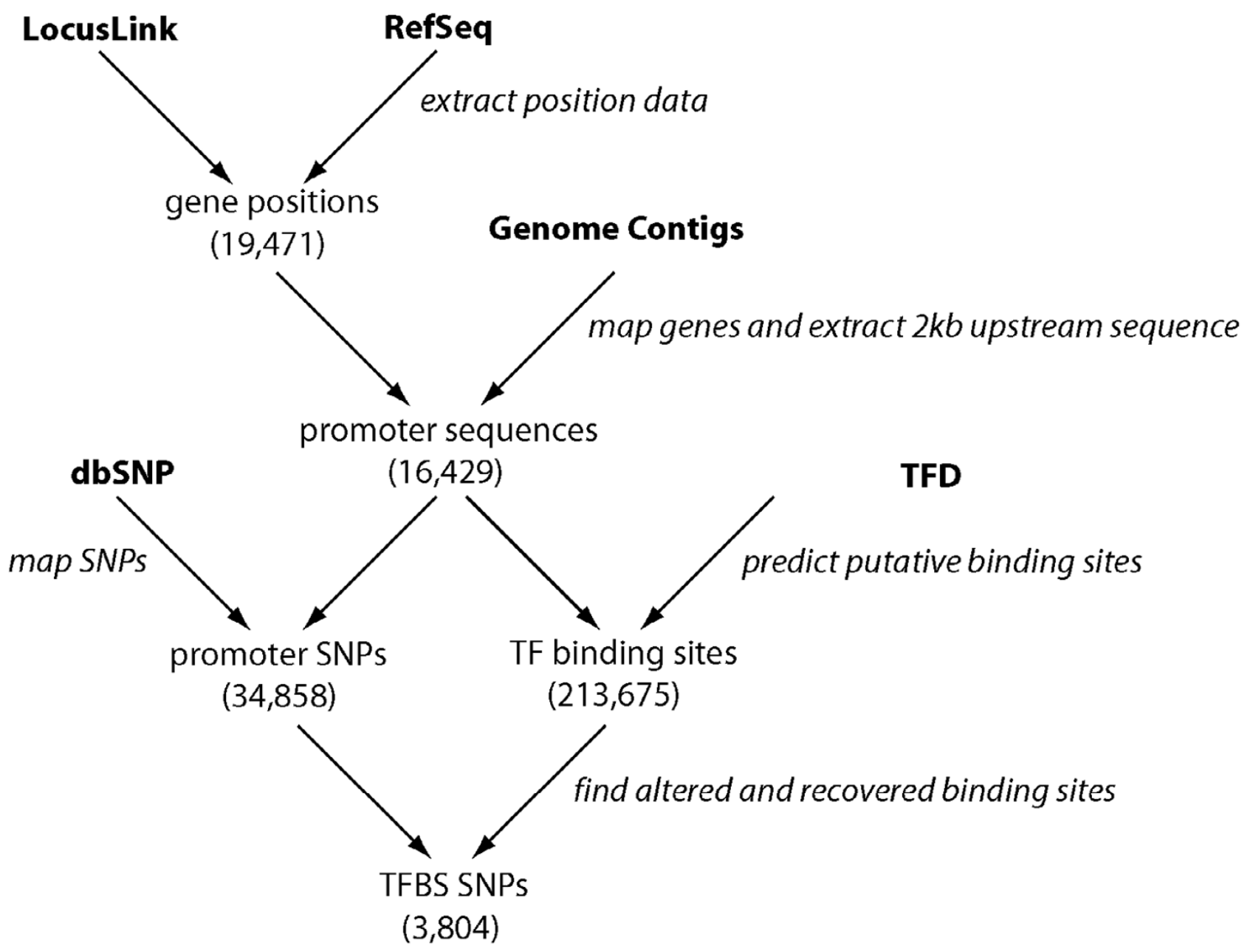

\section{Figure 4}

Flow chart of analysis steps used to generate data set. A high-level view of the methods used to generate the data sets used in this study. Canonical data sources are shown in bold, and general steps are shown in italics. Parenthetical numbers indicate the number of unique data elements in the data set.

\section{Data redundancy of transcription factor binding sites} Transcription factor binding site sequences were acquired from the Transcription Factor Database (TFD) [20]. The database contains more than three thousand mammalian binding site sequence entries encoded using the nomenclature of International Union of Pure and Applied Chemistry (IUPAC) [21]. Inspection of the sequence data revealed that some sequences differ at only one or two nucleotides, and some are subsequences of other sequences. To evaluate possible data redundancy, the binding site sequences were mutually compared and the sequence pairs were grouped into three categories: exact coverage (a complete match), partial coverage (one is a subsequence of the other) and single nucleotide difference (differing at an internal nucleotide). Binding sites in the partial coverage and single nucleotide difference categories were merged.

\section{Transcription factor binding site identification and distribution in gene promoters}

Transcription factor binding sites were predicted using a suffix tree algorithm $[22,23]$. The IUPAC codes were fully expanded and placed into a suffix tree, which allows the exact promoter sequence string to be searched against the 
binding site motifs while preserving the degeneracy of the binding sites. The probability of the binding site motif appearing by chance was computed using an expectation value given by the equation $F=\prod_{i=1}^{n} \frac{k}{4}$, where $k$ is the number of possible nucleotides at position $i$.

The distribution of binding sites in gene promoters was examined in order to identify the relation between occurrence of binding sites and location in gene promoter regions. Calculations were performed only upon the full length ( $2 \mathrm{~K} \mathrm{bp}$ ) promoters sequence. A $200 \mathrm{bp}$ portion of the gene subsequence just downstream of the transcriptional start site was also included with the promoter sequences. Expectation values of 0.1 and 0.01 were used as the control for binding site significance.

To evaluate the chance appearance of transcription factor binding sites, we computed their occurrences in two HMM-generated random datasets that simulate the promoter sequence range of $[-2000,-1]$. The first dataset was created by assigning equal an emission probability to all four nucleotides at each position, while for the second dataset the emission probability of nucleotide was determined according to the observed nucleotide probabilities from the real promoter dataset. Both simulated data sets contained the same number of sequences as the real promoter set.

\section{SNP distribution in putative binding sites}

Integrity of transcription factor binding sites was analyzed by comparing the SNP distributions within and outside the putative transcription binding sites. The transcription factor binding site data set contained both the binding sites directly identified from the canonical sequence, and the binding sites "recovered" by substituting previously identified SNP into the sequence.

\section{SNP distribution in experimentally derived binding sites} Experimentally derived transcription factor binding sites were retrieved from TRANSFAC database 6.0 [24], the eukaryotic transcription factors database. To evaluate whether SNPs occur in the experimentally derived TF binding sites, we linked the predicted TF binding sites with the binding sites in TRANSFAC using RefSeq literature references. The initial linkage was then confirmed using binding site sequence patterns, site locations and the order of the binding sites in gene promoter.

\section{Authors' contributions}

YG conceived of the study and carried out the coding and statistical analysis. DCJ participated in its design and coordination, and helped draft the manuscript.

\section{References}

I. Smigielski EM, Sirotkin K, Ward M, Sherry ST: dbSNP: a database of single nucleotide polymorphisms. Nucleic Acids Res 2000, 28:352-355.

2. Zimdahl H, Nyakatura G, Brandt $P$, Schulz $H$, Hummel $O$, Fartmann $B$, Brett D, Droege M, Monti J, Lee YA, Sun Y, Zhao S, Winter EE, Ponting CP, Chen Y, Kasprzyk A, Birney E, Ganten D, Hubner N: A SNP map of the rat genome generated from cDNA sequences. Science 2004, 303:807.

3. Zhu YL, Song QJ, Hyten DL, Van Tassell CP, Matukumalli LK, Grimm DR, Hyatt SM, Fickus EW, Young ND, Cregan PB: Single-nucleotide polymorphisms in soybean. Genetics 2003, I 63: I I 23-I I 34.

4. Collins FS, Morgan M, Patrinos A: The Human Genome Project: lessons from large-scale biology. Science 2003, 300:286-290.

5. Sachidanandam R, Weissman D, Schmidt SC, Kakol JM, Stein LD, Marth G, Sherry S, Mullikin JC, Mortimore BJ, Willey DL, Hunt SE, Cole CG, Coggill PC, Rice CM, Ning Z, Rogers J, Bentley DR, Kwok PY, Mardis ER, Yeh RT, Schultz B, Cook L, Davenport R, Dante M, Fulton L, Hillier L, Waterston RH, McPherson JD, Gilman B, Schaffner S, Van Etten WJ, Reich D, Higgins J, Daly MJ, Blumenstiel B, Baldwin J, Stange-Thomann N, Zody MC, Linton L, Lander ES, Altshuler D: A map of human genome sequence variation containing $\mathbf{1 . 4 2}$ million single nucleotide polymorphisms. Nature 2001, 409:928-933.

6. Sherry ST, Ward M, Sirotkin K: dbSNP-database for single nucleotide polymorphisms and other classes of minor genetic variation. Genome Res 1999, 9:677-679.

7. Ramensky V, Bork P, Sunyaev S: Human non-synonymous SNPs: server and survey. Nucleic Acids Res 2002, 30:3894-3900.

8. Clifford RJ, Edmonson MN, Nguyen C, Buetow KH: Large-scale analysis of non-synonymous coding region single nucleotide polymorphisms. Bioinformatics 2004, 20:1006-1014.

9. Ober C, Aldrich CL, Chervoneva I, Billstrand C, Rahimov F, Gray HL, Hyslop T: Variation in the HLA-G promoter region influences miscarriage rates. Am J Hum Genet 2003, 72: I425-I435.

10. Kiewitz C, Tummler B: Similar profile of single nucleotide substitution types in bacteria and human genetic disease. Genome Letters 2002, I: I I I-I |4.

II. Kel-Margoulis OV, Tchekmenev D, Kel AE, Goessling E, Hornischer K, Lewicki-Potapov B, Wingender E: Composition-sensitive analysis of the human genome for regulatory signals. In Silico Biol 2003, 3:|45-I7|.

12. Benson DA, Karsch-Mizrachi I, Lipman DJ, Ostell J, Wheeler DL: GenBank. Nucleic Acids Res 2003, 3 I:23-27.

13. Wong HC, Schnepf HE, Whiteley HR: Transcriptional and translational start sites for the Bacillus thuringiensis crystal protein gene. J Biol Chem 1983, 258:1960-1967.

14. Selvanayagam CS, Tsai SY, Tsai MJ, Selvanayagam P, Saunders GF: Multiple origins of transcription for the human placental lactogen genes. J Biol Chem I984, 259: | 4642-I4646.

15. Wu T, Ikezono T, Angus CW, Shelhamer JH: Characterization of the promoter for the human $85 \mathrm{kDa}$ cytosolic phospholipase A2 gene. Nucleic Acids Res 1994, 22:5093-5098.

16. Cavin Perier R, Junier T, Bucher P: The Eukaryotic Promoter Database EPD. Nucleic Acids Res 1998, 26:353-357.

17. Suzuki Y, Yamashita R, Nakai K, Sugano S: DBTSS: DataBase of human Transcriptional Start Sites and full-length cDNAs. Nucleic Acids Res 2002, 30:328-33I.

18. Suzuki Y, Yoshitomo-Nakagawa K, Maruyama K, Suyama A, Sugano S: Construction and characterization of a full length-enriched and a 5'-end-enriched cDNA library. Gene 1997, 200:149-156.

19. Balasubramanian S, Harrison P, Hegyi H, Bertone P, Luscombe N, Echols N, McGarvey P, Zhang Z, Gerstein M: SNPs on human chromosomes 21 and 22 -- analysis in terms of protein features and pseudogenes. Pharmacogenomics 2002, 3:393-402.

20. Ghosh D: Object-oriented transcription factors database (ooTFD). Nucleic Acids Res 2000, 28:308-310.

21. Dybkaer R: International Union of Pure and Applied Chemistry and International Federation of Clinical Chemistry. IUPAC Section of Clinical Chemistry; Commission on Quantities and Units in Clinical Chemistry and IFCC Committee on Standards, Expert Panel on Quantities and Units; Approved Recommendation (1978). Quantities and Units in Clinical Chemistry. Clin Chim Acta 1979, 96:I57F-I83F.

22. Weiner P: Linear pattern matching algorithms. I973:I-II. 
23. McCreight EM: A space-economical suffix tree construction algorithm. Journal of Algorithms 1976, 23:262-272.

24. Wingender E, Chen X, Fricke E, Geffers R, Hehl R, Liebich I, Krull M, Matys V, Michael H, Ohnhauser R, Pruss M, Schacherer F, Thiele S, Urbach S: The TRANSFAC system on gene expression regulation. Nucleic Acids Res 2001, 29:28I-283.

25. Paulweber B, Levy-Wilson B: The mechanisms by which a human apolipoprotein $B$ gene enhancer and reducer interact with the promoter are different in cultured cells of hepatic and intestinal origin. J Biol Chem 199|, 266:24I6I-24I68.

26. Metzger S, Halaas JL, Breslow JL, Sladek FM: Orphan receptor HNF-4 and bZip protein C/EBP alpha bind to overlapping regions of the apolipoprotein $B$ gene promoter and synergistically activate transcription. J Biol Chem 1993, 268: $1683 \mid-16838$.

27. Fisch TM, Prywes R, Roeder RG: c-fos sequence necessary for basal expression and induction by epidermal growth factor, 12-O-tetradecanoyl phorbol-13-acetate and the calcium ionophore. Mol Cell Biol 1987, 7:3490-3502.

28. Bergemann AD, Johnson EM: The HeLa Pur factor binds singlestranded DNA at a specific element conserved in gene flanking regions and origins of DNA replication. Mol Cell Biol 1992, 12:1257-1265.

29. Postel EH, Berberich SJ, Flint SJ, Ferrone CA: Human c-myc transcription factor PuF identified as $\mathrm{nm} 23-\mathrm{H} 2$ nucleoside diphosphate kinase, a candidate suppressor of tumor metastasis. Science 1993, 26 I:478-480.

30. Englander EW, Wilson SH: Protein binding elements in the human beta-polymerase promoter. Nucleic Acids Res 1990, 18:919-928.

31. Kedar PS, Widen SG, Englander EW, Fornace AJJ, Wilson SH: The ATF/CREB transcription factor-binding site in the polymerase beta promoter mediates the positive effect of $\mathrm{N}$-methylN'-nitro-N-nitrosoguanidine on transcription. Proc Natl Acad Sci USA I99I, 88:3729-3733.

32. Wang TC, Brand SJ: Islet cell-specific regulatory domain in the gastrin promoter contains adjacent positive and negative DNA elements. J Biol Chem 1990, 265:8908-89I4.

33. Leclerc S, Xie BX, Roy R, Govindan MV: Purification of a human glucocorticoid receptor gene promoter-binding protein. Production of polyclonal antibodies against the purified factor. J Biol Chem 1991, 266:87II-87I9.

34. Ray A, LaForge KS, Sehgal PB: On the mechanism for efficient repression of the interleukin-6 promoter by glucocorticoids: enhancer, TATA box, and RNA start site (Inr motif) occlusion. Mol Cell Biol 1990, 10:5736-5746.

35. Araki E, Murakami T, Shirotani T, Kanai F, Shinohara Y, Shimada F, Mori M, Shichiri M, Ebina Y: A cluster of four Sp I binding sites required for efficient expression of the human insulin receptor gene. J Biol Chem I991, 266:3944-3948.

36. Hasegawa SL, Boss JM: Two B cell factors bind the HLA-DRA $X$ box region and recognize different subsets of HLA class II promoters. Nucleic Acids Res 1991, 19:6269-6276.

37. Shakhov AN, Collart MA, Vassalli P, Nedospasov SA, Jongeneel CV: Kappa B-type enhancers are involved in lipopolysaccharidemediated transcriptional activation of the tumor necrosis factor alpha gene in primary macrophages. J Exp Med 1990, | $71: 35-47$
Publish with Biomed Central and every scientist can read your work free of charge

"BioMed Central will be the most significant development for disseminating the results of biomedical research in our lifetime. "

Sir Paul Nurse, Cancer Research UK

Your research papers will be:

- available free of charge to the entire biomedical community

- peer reviewed and published immediately upon acceptance

- cited in PubMed and archived on PubMed Central

- yours - you keep the copyright

Submit your manuscript here:

http://www.biomedcentral.com/info/publishing_adv.asp
BioMedcentral 